\title{
Log-periodic Oscillations for Biased Diffusion on Random Lattice
}

\author{
Dietrich Stauffer ${ }^{1}$ and Didier Sornette ${ }^{2}$ \\ ${ }^{1}$ Institute for Theoretical Physics, Cologne University, 50923 Köln, Germany \\ e-mail: stauffer@thp.uni-koeln.de \\ ${ }^{2}$ Department of Earth and Space Science and Institute of Geophysics and Planetary \\ Physics, University of California, Los Angeles, California 90095, USA and Laboratoire \\ de Physique de la Matière Condensée, CNRS UMR6622 and Université de Nice- \\ Sophia Antipolis, Faculté des Sciences, B.P. 7106108 NICE Cedex 2, France \\ e-mail: sornette@cyclop.ess.ucla.edu
}

\begin{abstract}
Random walks with a fixed bias direction on randomly diluted cubic lattices far above the percolation threshold exhibit log-periodic oscillations in the effective exponent versus time. A scaling argument accounts for the numerical results in the limit of large biases and small dilution and shows the importance of the interplay of these two ingredients in the generation of the log-periodicity. These results show that log-periodicity is the dominant effect compared to previous predictions of and reports on anomalous diffusion.
\end{abstract}

Diffusion on percolating lattices has a long history [1-4]. Here the sites of a large lattice are randomly initialized as being accessible, with probability $p$, or forbidden, with probability $1-p$; and random walker diffuse on the accessible sites only. In biased diffusion $[5,6]$, with a probability $B$ the random walk moves in one fixed direction, while with probability $1-B$ it moves as usual to one randomly selected nearest neighbor; in both cases the move is allowed only if the neighbor is accessible. This fixed bias corresponds to an external electric field and is different from the bias with changing direction depending on the flow through the random network [7].

Numerical searches for a possible phase transition as a function of the bias $B$ were hampered by strong variations of the effective exponent $k$ in

$$
<r^{2}>\propto t^{k}
$$

as a function of time $t$, for the mean square displacement $r$. In particular, Seifert and Suessenbach [8] found smooth variations, if $k$ was plotted versus the logarithm of time. Log-periodic oscillations were predicted before for a special one-dimensional diffusion problem [9] while ref.8 investigated three dimensions.

In the meantime, both computer technology and theoretical understanding have advanced appreciably. Log-periodic oscillations were seen in many cases, as reviewed recently [10], including the stock market crashes on Wall Street in 1929 and 1987 [11] and in 1997 [12]. The physical mechanism for log-periodicity obtained in [9] is the exponential dependence of the trapping time on the length of the traps that are multiples of the lattice mesh size. The resulting intermittent random walk is thus punctuated by the successive encounters with larger and larger clusters of trapping sites. For strong bias $B$ in three dimensions, we might expect a somewhat similar 
mechanism as discussed below. We were thus motivated to repeat the work of ref.[8] for longer times, in search for periodicities like $\sin (\log (t))$.

Each of the 512 processors of the Cray-T3E deals with about 3 million steps per second, roughly the same speed as the supercomputer CDC Cyber 205 used in ref.[8]. We could also simulate larger simple-cubic lattices $(301 \times 301 \times 301)$ even though we wasted a whole 32-bit word for each lattice site (instead of one bit only) to simplify and accelerate the program (93 Fortran lines, available from the first author). We averaged over about 500 lattices, with 1000 walkers on each for short times and 3 for long times up to $4 \times 10^{10}$.

If the bias equals $B=1$, the motion is one-dimensional, and thus because of ref.[9] the chances for log-periodic oscillations are best for high $B$. Indeed, ref.[8] found clearer oscillations for $B=0.99$ than for $B=0.9$. Fig.1 shows our results for $B=0.98$ giving very clearly three maxima in $k v s . \log (t)$ near $t=10^{4}, 210^{6}, 410^{8}$. Fig. 2 shows our results for $B=0.999$ giving two maxima in $k v s$. $\log (t)$ near $t=10^{5}, 10^{9}$. Fig. 3 shows our results for $B=0.95$ giving many more maxima but with much smaller amplitude which are thus harder to distinguish from noise. As a function of the bias $0.9<B<0.999$ at fixed concentration $p=0.725$ we found slower oscillations for larger $B$, while for smaller $B$ they became more rapid. For smaller $B$ 's, the oscillations become masked by the random fluctuations. We could probably attempt to measure them by a suitable averaging procedure $[10,13]$ and leave this for the future.

Table 1: The table summarizes our estimates for the scaling factors $\lambda$ defined as the ratio of the times of the successive maxima.

\begin{tabular}{|c|c|c|}
\hline $\mathrm{B}$ & $\lambda$ & $(1+5 \mathrm{~B}) /(1-\mathrm{B})$ \\
\hline 0.90 & $10 \pm 5$ & 55 \\
\hline 0.95 & $15 \pm 5$ & 110 \\
\hline 0.98 & $200 \pm 100$ & 300 \\
\hline 0.99 & $10^{3}$ & $610^{2}$ \\
\hline 0.999 & $10^{4}$ & $610^{3}$ \\
\hline
\end{tabular}

We now present the scaling argument proposed to account for these results, that should be correct in the limit $B \rightarrow 1$ and $1-p \rightarrow 0$ but non zero. We expect its domain of validity to be much broader than these limits would indicate, in a way similar to what we found for the scaling argument developed in [14] (see also [10]) to retrieve the exact analytical results derived in [9]. The mechanism is best understood by noticing that the biased random walk along $x$ is the same as an unbiased random walk in the presence of a linearly decreasing potential

$$
V(x)=-b x
$$

where the slope $b$ is such that the ratio of the forward over backward motion rate parallel to the bias is correctly evaluated. In the definition involving $B$, the forward 
rate is $\frac{1-B}{6}+B=\frac{1+5 B}{6}$ (the unbiased contribution plus the bias) and the backward rate is $\frac{1-B}{6}$. Equating their ratio to $\exp \left[\begin{array}{ll}\beta & b\end{array}\right]$ yields $b$ in (11), where $\beta$ is the inverse temperature in the proposed analogy:

$$
b=\frac{1}{\beta} \log \frac{1+5 B}{1-B} .
$$

$b \propto B$ for small $B$ and $b \propto \log \frac{1}{1-B}$ for $B$ close to one.

As the random walker makes its way through the system, it will eventually encounter a local well, defined by a connected peninsula surrounded by empty sites (this can also be called a dead end) such that it has to retrace its steps backward to escape. Call $n$ the depth of this well. The typical time of residence of the walker in this well (trapping time) is proportional to the Arrhenius factor $\exp [\beta b n]$. This is the mechanism that converts a linear increase (in $n$ ) into an exponential increase in trapping times and thus produces log-periodic oscillations. Indeed, at short times, it is more probable that the smallest well be first encountered. This is due to the fact that such trapping wells occur with small probability (see below), denoted $P_{n}$. The smallest wells are of depth $n=1$ and lead to a trapping time $\propto \exp [\beta \quad b]=\frac{1+5 B}{1-B}$. The next most severe trapping wells are of depth $n=2$ and lead to a typical trapping time $\propto \exp [2 \beta b]=\left[\frac{1+5 B}{1-B}\right]^{2}$. And so on. We see a hierarchy of time scales $\left[\frac{1+5 B}{1-B}\right]^{n}$ resulting from the succession of worst encountered trapping wells. Note that after it has encountered a well of depth $n$, the walker will in general encounter several wells of the same or smaller depths before being trapped in a well of depth $n+1$. This does not destroy the argument because only the worst trapping well encountered until now dominates the waiting times and thus the log-periodic modulations. This is completely similar to what was found in the one-dimensional case $[9,10]$. Note that we also predict that only a finite number $n_{\max }$ of oscillations will be observed in a finite system of linear size $L$. This simply stems from the finiteness of the deepest well $n_{\max }$ in a finite system, given by the condition $P_{n_{\max }} L^{d} \sim 1$ (J. Machta and A. Aharony, priv.comm.). If $P_{n}$ is exponentially decreasing with $n$, we see that $n_{\max } \sim \log L$, while it is even smaller $n_{\max } \sim[\log L]^{\frac{1}{d-1}}$ according to our suggestion below. This size effect has been verified qualitatively for $B=0.98$, with the same concentration $p=0.725$ and sizes $L=51,21,11$ and 5 .

Our theory teaches us that only the depth of the wells control the leading behavior of the trapping time scales. Their transverse sizes bring in only subleading power law corrections to the exponential Arrhenius dependence. This is the fundamental reason for the existence and robustness of the observed log-periodic oscillations, notwithstanding the many random configurations that the trapping wells can take. Notice that, as in all other known cases [10], the log-periodic oscillations rely on the existence of an ultraviolet (or infrared) cut-off, here the mesh size.

We have reported in the last column of table 1 the value of the scaling factor

$$
\lambda \equiv \frac{t_{n+1}}{t_{n}}=\frac{1+5 B}{1-B}
$$

derived from (2). The agreement is quite good for the largest bias $B \rightarrow 1$ for which the first smallest wells dominate completely the dynamics. For smaller $B$ 's, corrections 
include the effect of all subleading time scales coming from all well shapes of a given depth. There is also a larger uncertainty in the determination of the scaling ratio $\lambda$ from the figures as the log-periodic oscillations are weaker and harder to distinguish from statistical noise. The small ratios seen for the smaller $B$ 's in the figures could also be higher harmonics.

This is all we need to account for the observed log-periodicity. Let us now briefly comment on the distribution $P_{n}$ of trapping well sizes of depth $n$. A trapping well of depth $n$ has a typical transverse size $n$ (isotropy). It can be constructed in a way similar to that leading to the distribution of finite cluster size above percolation. In this latter case, a finite cluster $s$ is constructed from the infinite incipient percolation network by introducing of the order of $s^{\frac{d-1}{d}}$ empty perimeter sites. This leads to the stretched exponential distribution $\exp \left[-a s^{1-1 / d}\right]$ known to be exact [15]. In order to construct a trapping well of depth $n$, one needs also to introduce of the order of $s^{1-1 / d}$ empty perimeter sites. This would predict the distribution $\exp \left[-a^{\prime} n^{d-1}\right]$, which decays faster than an exponential in dimensions larger than two. This is in contrast to [6] which used an exponential distribution to predict a transition from drift to no drift (vanishing velocity) above a critical value of the bias $B$. Our results show that this effect is very hard to detect numerically if true and that the main effect of the trapping wells is by far in creating a hierarchy of time scales $t_{n}$ given by (3) leading to log-periodic oscillations that completely dominates the dynamics.

To summarize, damped oscillations, periodic in the logarithm of the time, have been discovered for strongly biased diffusion on disordered three-dimensional lattices; these log-periodic oscillations stem from an Arrhenius exponentiation of a discrete spectrum of trapping well depths, leading to a discrete hierarchy of time scales. Similar effects were found for concentration $p=0.5$, while for bias $B=0.5$ no oscillations were seen. Our result underlines the importance of accounting for intermittent distortions that will bias the determination of exponents whenever log-periodicity occurs. We note that a similar difficulty for determining the fractal dimension of fractal DLA clusters in the past has been attributed to the existence of log-periodic modulation of the apparent exponent [16].

We are indebted to M. Barma and D. Dhar for pointing out an error in a previous version and for useful correspondences. We thank the Les Houches Spring School 1997 "Scaling and Beyond" [17] as well as L. Schäfer and his group at Essen University and J. Machta and A. Aharony for crucial questions and suggestions. The simulations were made at HLR Jülich in several thousand processor hours. 
[1] P. G. de Gennes, La Recherche 7, 916 (1976).

[2] C. D. Mitescu H. Ottavi and J. Roussenq, AIP Conference Proceedings 40, 377 (1978)

[3] Y. Gefen, A. Aharony, S. Alexander; D. Ben-Avraham and S. Havlin, J. Phys. A 15, L 691 (1992).

[4] S. Havlin and D. Ben Avraham, Adv. Phys. 36, 395 (1987)

[5] R.B.Pandey, Phys.Rev. B 30, 489 (1984); H. Böttger and V. V. Bryskin, Phys. Stat. Sol. (b) 113, 9 (1982).

[6] M. Barma and D. Dhar, J. Phys. C 16, 1451 (1983).

[7] A.Bunde, S. Havlin and H.E.Roman, Phys.Rev. A 42, 6274 (1990) and earlier papers of this group cited there.

[8] E. Seifert and M. Suessenbach, J.Phys.A 17, L 703 (1994)

[9] J. Bernasconi and W.R. Schneider, J.Phys. A 15, L 729 (1982)

[10] D.Sornette, Physics Reports, in press (http://xxx.lanl.gov/abs/cond-mat/9707012)

[11] D. Sornette, A. Johansen and J.-P. Bouchaud, J.Phys.I France 6, 167 (1996); D.

Sornette and A. Johansen, Physica A 245, 411 (1997)

[12] Prediction of the stock market turmoil at the end of october 1997, based on an unpublished extension of the theory, have been formally issued ex-ante on september 17, 1997, to the French office for the protection of proprietary softwares and inventions under number registration 94781. In addition, a trading strategy has been devised using put options in order to provide an experimental test of the theory. A $400 \%$ profit has been obtained in a two week period covering the mini-crash of october 31, 1997. The proof of this profit is available from a Merrill Lynch client cash management account released in november 1997. See also H. Dupuis, "Un krach avant novembre", Tendances, 18. September 1997, page 26, from the work of N. Vandewalle, A.Minguet, P.Boveroux, and M. Ausloos using the same type of log-periodic signals.

[13] A. Johansen and D. Sornette, "Canonical averaging" for log-periodicity, preprint [14] H. Saleur, C.G. Sammis and D. Sornette, J. Geophys. Res. 101, 17661 (1996)

[15] H. Kunz and Souillard, B., J. Stat. Phys. 19, 77 (1978).

[16] D. Sornette, A. Johansen, A. Arnéodo, J.-F. Muzy and H. Saleur, Phys. Rev. Lett. 76, 251 (1996).

[17] B. Dubrulle, F. Graner and D. Sornette, eds., Scale invariance and beyond, Proceedings of Scale invariance and Beyond, Springer, Heidelberg, 1997.

Fig.1: Effective exponent $k=\mathrm{d} \log r^{2} / \mathrm{d} \log t$ versus time $t$ for $B=0.98$. (combination of different runs with different statistics).

Fig.2: Same as Fig.1 for $B=0.999$.

Fig.3: Same as Fig.1 for $B=0.95$. 